\title{
(6) OPEN ACCESS \\ Doxycycline in early CJD: a double-blinded randomised phase II and observational study
}

\author{
Daniela Varges, ${ }^{1}$ Henrike Manthey, ${ }^{1}$ Uta Heinemann, ${ }^{1}$ Claudia Ponto, ${ }^{1}$ \\ Matthias Schmitz, ${ }^{1}$ Walter J Schulz-Schaeffer, ${ }^{2}$ Anna Krasnianski, ${ }^{1}$ Maren Breithaupt, ${ }_{1}^{1}$ \\ Fabian Fincke, ${ }_{1}^{1}$ Katharina Kramer, ${ }^{3}$ Tim Friede, $^{3}$ Inga Zerr $^{1}$
}

\begin{abstract}
${ }^{1}$ Department of Neurology, National Reference Center for TSE Surveillance, University Medical Center Goettingen, Göttingen, Germany ${ }^{2}$ Department of Neuropathology, University Medical Center Goettingen, Göttingen, Germany

${ }^{3}$ Department of Medical Statistics, University Medical Center Goettingen, Göttingen, Germany
\end{abstract}

\section{Correspondence to}

Professor Inga Zerr, National Reference Center for TSE Surveillance, Clinical Dementia Center, Department of Neurology, University Medical Center, Robert-Koch Str. 40, Göttingen D-37075, Germany; ingazerr@med. uni-goettingen.de

Received 14 March 2016 Revised 15 June 2016 Accepted 21 June 2016 Published Online First 2 November 2016

\section{SLinked}

- http://dx.doi.org/10.1136/ jnnp-2016-314578

\section{CrossMark}

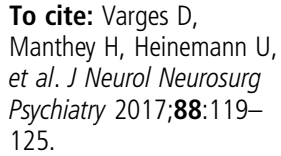

\begin{abstract}
Objectives The main objective of the present study is to study the therapeutic efficiency of doxycycline in a double-blinded randomised phase II study in a cohort of patients with sporadic Creutzfeldt-Jakob disease (sCJD). Methods From the National Reference Center of TSE Surveillance in Germany, patients with probable or definite SCJD were recruited for a double-blinded randomised study with oral doxycycline (EudraCT 2006-003934-14). In addition, we analysed the data from patients with CJD who received compassionate treatment with doxycycline in a separate group. Potential factors which influence survival such as age at onset, gender, codon 129 polymorphism and cognitive functions were evaluated. The primary outcome measure was survival.
\end{abstract}

Results Group 1: in the double-blinded randomised phase II study, 7 patients in the treatment group were compared with 5 controls. Group 2: 55 patients with SCJD treated with oral doxycycline were analysed and compared with 33 controls by a stratified propensity score applied to a Cox proportional hazard analysis. The results of both studies were combined by means of a random-effects meta-analysis. A slight increase in survival time in the doxycycline treatment group was observed ( $p=0.049, H R=0.63(95 \% \mathrm{Cl} 0.402$ to 0.999)).

Conclusions On the basis of our studies, a larger trial of doxycycline should be performed in persons in the earliest stages of CJD.

Trial registration number EudraCT 2006-003934-14; Results.

\section{INTRODUCTION}

Numerous drugs have been tried and have failed in in vivo models of sporadic Creutzfeldt-Jakob disease (sCJD) ${ }^{1-4}$ Enrolment of patients with CJD in randomised trials poses specific problems, such as refusal to give informed consent due to the risk of receiving a placebo for a fatal disease and patients' preference for the effective substance, ${ }^{5}$ and most studies are limited to the low disease incidence (table 1).

Doxycycline was reported as an antiprion agent in in vitro and animal models. ${ }^{6}{ }^{7}$ Recently, the results of a phase 2 trial failed to demonstrate doxycycline efficacy. ${ }^{8}$ However, the study included mainly patients in advanced disease stages, as evidenced by the inclusion criteria and the clinical data, such as disease duration more than 5 months at enrolment in 39\% and high periodic sharp wave complexes (PSWC) rate in the EEG (normally at late stages). Therefore, we hypothesised that the enrolment of severely impaired patients could be the reason for the resultant lack of efficacy. Here, we analyse patients at an early stage of disease (defined by neuropsychological tests) in a doubleblinded prospective study. In addition, we analysed data from patients who received doxycycline as compassionate treatment, who were matched to a control group which was selected by known predictors for disease aggressiveness in CJD (age at onset, gender and codon 129). Then we combined data from an observational study and that obtained from the randomised trial in a meta-analytic framework.

\section{PATIENTS AND METHODS}

Our study is designed to provide class IV evidence; its primary research aim is to evaluate the potential therapeutic effect of doxycycline as an antiprion drug in patients with sCJD.

\section{Patients}

Patients with suspected human prion diseases were reported to the National Reference Center for TSE Surveillance in Göttingen, Germany, and examined on site by a study physician. Cerebrospinal fluid (CSF), blood samples and copies of important diagnostic tests (MRI, EEG, laboratory tests) were obtained. Patients with suspected CJD were classified according to established diagnostic criteria. ${ }^{9} 10$ The date of disease onset was determined by interview with the relatives. If the exact date could not be stated, relatives were asked for the month of onset and the 15th of that month was used as the onset date for further calculations. The investigator determining this date was blinded to treatment.

Between March 2010 and September 2012, females and males between 18 and 80 years of age with diagnoses of probable or definite $\mathrm{sCJD}^{9} 10$ and a disease duration of $<24$ months were included in this randomised trial. Further inclusion criteria were a Mini-Mental State Examination (MMSE) score $>6$ points or Barthel index scores $\geq 20 \%$, cMRI within the past 6 months, EEG within the past 3 months and no contraindications for doxycycline. Patients with PRNP mutations were excluded. The study was conducted at the University of Göttingen, Germany, and was approved by the Ethics Committee of Göttingen (reference number: 26/7/06). Written informed consent was obtained from the patients and/or 
Table 1 Results on therapeutic trials on patients with sporadic Creutzfeldt-Jakob disease

\begin{tabular}{|c|c|c|c|c|c|}
\hline Active substance & Reference & Patients (n) & Study design & Prolongation of survival time & Further results \\
\hline \multirow[t]{2}{*}{ Amantadine } & Terzano et $a l^{24}$ & 4 & Observational & No & Increased alertness \\
\hline & Neri et $a l^{25}$ & 8 & Observational & No & Increased alertness \\
\hline Flupirtine & Otto et $a l^{11}$ & 13 & Randomised controlled clinical trials & No & Less cognitive decline \\
\hline \multirow[t]{2}{*}{ Pentosan-polyphosphate } & Bone et $a l^{26}$ & 7 & Observational & Possibly extended & \\
\hline & Tsuboi et $\left.a\right|^{27}$ & 11 & Observational & Possibly extended & \\
\hline \multirow[t]{3}{*}{ Quinacrine } & Haik et $a l^{28}$ & 32 & Case-control & No & \\
\hline & Collinge et $a l^{17}$ & 40 & Observational & No & \\
\hline & Geschwind et $a l^{18}$ & 23 & Observational & No & Less cognitive decline \\
\hline Doxycycline & Haik et $a l^{8}$ & 62 & Randomised controlled clinical trials & No & \\
\hline
\end{tabular}

their legally authorised representatives. Patients were randomly assigned (ratio 1:1) to receive either doxycycline or placebo. The randomisation list and the study medication were administered by the Charité, Universitätsmedizin, Berlin, Germany. The patients in the therapy group received oral doxycycline at a dose of $100 \mathrm{mg}$ per day. After the baseline visit (BL) and the first visit (visit 1), the patients were visited every 2 months during the first 6 months (visits 2-4) and then every 3 months. Additionally, the caregivers were interviewed by telephone every 2 weeks to evaluate the disease progression based on a standardised questionnaire.

Compassionate treatment data: Patients with suspected sCJD were seen by a physician at the time of diagnosis. Standardised clinical examination with special emphasis on CJD-specific symptoms and standardised scales to measure clinical presentation (Barthel index) and cognitive decline (MMSE) was performed.

Compassionate treatment with doxycycline (100 mg daily dose) was initiated by the family doctor. Follow-up clinical examination in the framework of surveillance studies was performed, including a clinical examination and neuropsychological test battery. Originally, 77 patients with sCJD were treated with doxycycline.

To avoid any bias, a control group was selected from historical data and included only patients who were diagnosed with probable or definite SCJD and were not treated with doxycycline and did not participate in a previous study with flupirtine. ${ }^{11}$

Only 33 patients were included as controls because of our strict selection criteria which were (1) diagnosis during the same time period as the group which received doxycycline; (2) all clinical data should be available; (3) genetic mutations in PRNP were excluded by sequence analysis and (4) the cohort was matched by age, gender and codon 129 genotype.

Since the baseline MMSE is an important prognostic factor, only patients with known baseline MMSE were considered for further analysis, resulting in a sample size of 88 (55 treated and 33 untreated) patients.

\section{Standard protocol approvals, registrations and patient} consents

The randomised, single-centre, double-blind, placebo-controlled phase II trial (EudraCT (2006-003934-14)) was approved by the local Ethics Committee of the University Medical School (26/7/2006 from 27.7.2007).

\section{Biochemical and neuropathological analyses}

CSF tests: CSF samples were obtained by lumbar puncture and the samples were subsequently frozen and stored in Eppendorf tubes at $-80^{\circ} \mathrm{C}$. Blood-contaminated CSF samples were excluded. Measurements of $\tau$ protein and neuron-specific enolase (NSE) by established ELISA methods and detection of 14-3-3 protein by means of western blot analysis were performed as described previously. ${ }^{12} 13$

Codon 129 genotyping: After isolation of genomic DNA from blood samples according to standard methods, analysis of the codon 129 genotype of PRNP was performed (after isolation of genomic DNA from blood samples according to standard methods ${ }^{14}$ ).

Outcome measures: The primary outcome measure was survival time. In the double-blinded randomised study, follow-up for survival starts with the randomisation (first application of study medication) and ends with date of death or end of the study, whichever occurs first. In the observational study, follow-up cannot be defined in the same way. Therefore, patients in the observational study are followed up for survival from disease onset. This start point is both a natural choice and also minimises the risk of bias when comparing patients treated with doxycycline to controls since the onset of symptoms is well defined in both groups as the date of the first interview with close relatives.

Other secondary measures included scores on cognitive and functional tests (Barthel index, MMSE, a neurological examination, CSF biomarkers (14-3-3 protein, NSE, $\tau$ protein), EEG and cerebral MRI).

\section{Statistical analyses}

Demographic and clinical characteristics of the cohorts were summarised by descriptive statistics and survival was defined as the primary end point. Survival times were analysed by means of Cox proportional hazard models with treatment (doxycycline vs control) as a factor. Treatment effects are reported in terms of HRs with $95 \%$ CIs and $\mathrm{p}$ values testing the null hypothesis of no effect of doxycycline on survival in comparison to untreated control or placebo, respectively. Analyses were adjusted for several prognostic factors including age at onset, gender, codon 129 genotype and baseline MMSE, by stratifying the Cox regression by terciles of the propensity scores. The stratification allows for differences in the baseline hazards across the three strata, but a common HR for the treatment effect could be assumed without a statistically significant loss of goodness of fit $(p=0.2703$, likelihood ratio test).

In order to combine the results of both groups, a random-effects meta-analysis was performed. A combined estimate of the $\log \mathrm{HR}$ for treatment was calculated using inversevariance weighting. Between-study heterogeneity was assessed by the so-called $\mathrm{I}^{2}$ measure. ${ }^{15}$ 
All reported $p$ values are two-sided and $p \leq 0.05$ is considered statistically significant. Statistical analyses were carried out using SAS (V.9.3 for Windows, Copyright 2002-2010 SAS Institute, Cary, North Carolina, USA) and the Review Manager (RevMan, V.5.2, Copenhagen: The Nordic Cochrane Centre, The Cochrane Collaboration, 2012).

\section{RESULTS}

The randomised, double-blinded, placebo-controlled trial Of 392 probable and patients with definite sCJD referred between March 2010 and September 2012, only 13 participants with SCJD agreed to participate in the randomised trial (figure 1).

The first patient was enrolled on 29 March 2010 and the last visit of the last patient was on 25 September 2012. Patients were randomised to active drug $(n=7)$ or placebo $(n=6)$. One patient who was randomised to the placebo group dropped out, so that 12 patients with sCJD were included in the reported survival analysis. The study demographics of the study population and the results of the MMSE and Barthel index, and the codon 129 polymorphism are given in table 2 .

The diagnoses of sCJD were supported by findings in the CSF, and by EEG and MRI, as given in table 2. The therapy started
105 days (3 months) from disease onset (mean). Mean treatment duration was 89 days. Cognitive deficits were quantified by MMSE (21 in the doxycycline group and 16 in the placebo group). The Barthel index score was slightly better in the placebo group.

The Cox proportional hazard analysis revealed no statistically significant prolongation in survival time in patients treated with doxycycline with an estimated HR of 0.841 (95\% CI $(0.238$ to 2.966)). The median survival time after initiation of doxycycline treatment was 95 days compared with 33 days for the control group. The prolonged survival times in doxycycline-treated patients with sCJD versus the non-treated patients with sCJD are given in figure $2 \mathrm{~A}$.

Secondary outcome measures focused on the quality of life. To analyse the doxycycline effect, we measured the time from disease onset to the onset of deficits in sphincter control and food intake, or the appearance of akinetic mutism. These events were registered slightly later in patients who received doxycycline (table 3). We performed a log-rank test in order to quantify these differences. All $\mathrm{p}$ values are about 0.9 and hence no significance could be observed.

The adverse event (AE) rate was $70 \%$ in the doxycycline group and $60 \%$ in the placebo group. The severe adverse event (SAE) rate was $40 \%$ in both groups. The recorded AEs/SAEs

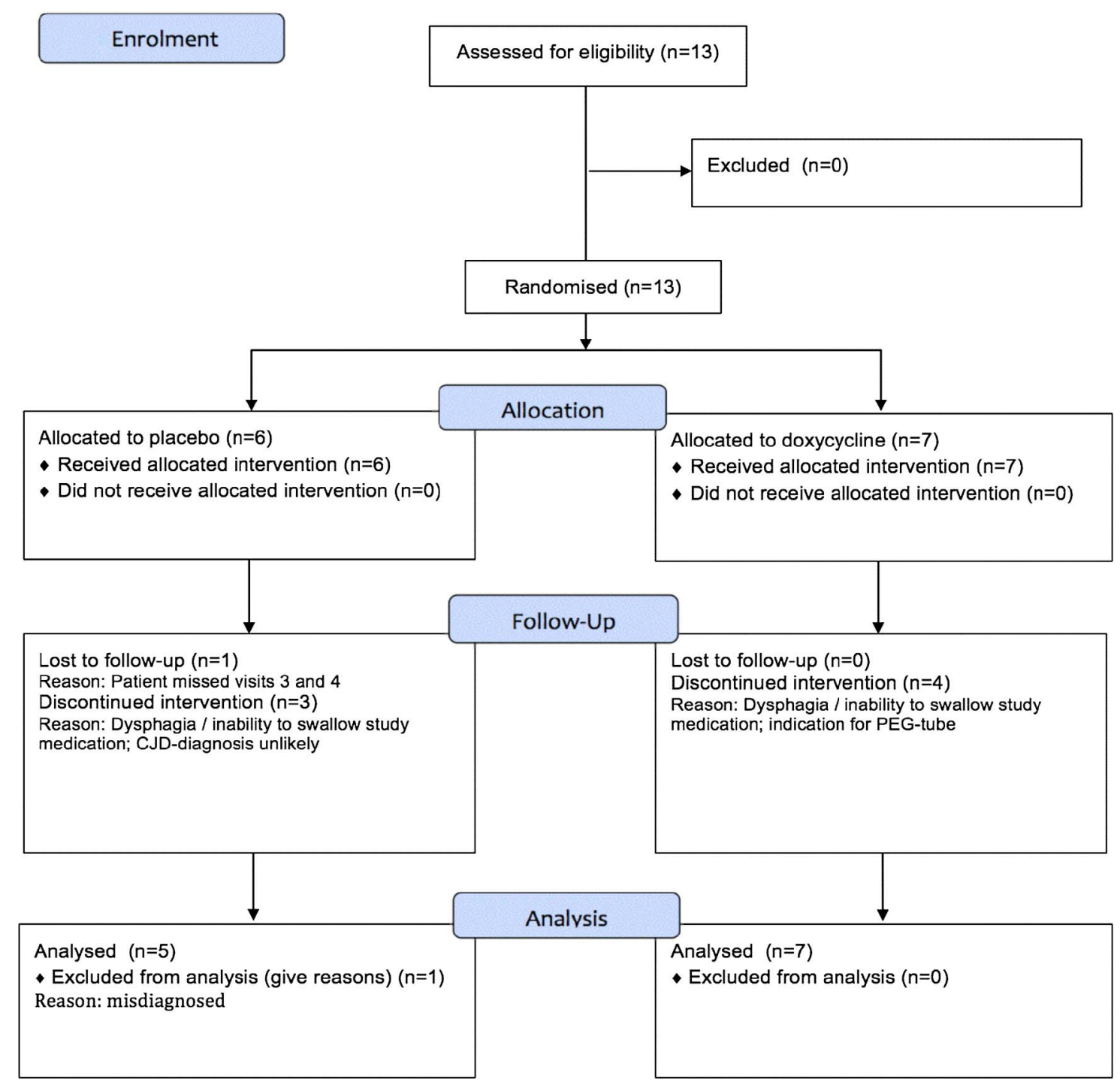

Figure 1 Overview clinical study. CJD, Creutzfeldt-Jakob disease; PEG, percutaneous endoscopic gastronomy. 
Neurodegeneration

Table 2 Clinical data and diagnostic findings of the study groups

\begin{tabular}{|c|c|c|c|c|}
\hline Characteristics/diagnostics & Double-blind doxycycline & Double-blind control & Observational doxycycline & Observational control \\
\hline \multirow[t]{2}{*}{ Female/male } & $2 / 5$ & $3 / 2$ & $24 / 31$ & $11 / 22$ \\
\hline & Median (IQR) & Median (IQR) & Median (IQR) & Median (IQR) \\
\hline Age at onset (years) & $58.0(56.0-66.0)$ & $72.0(55.0-73.0)$ & $62.1(57.3-69.9)$ & $65.0(56.0-68.0)$ \\
\hline Days from onset to start of (placebo) therapy & $95(75-145)$ & $152(99-701)$ & $138(92-212)$ & - \\
\hline Days of doxycycline/placebo therapy & $61(11-156)$ & $34(1-439)$ & $88(48-148)$ & - \\
\hline MMSE points & $21(21-25)$ & $16(8-25)$ & $20(12-24)$ & $12(0-21)$ \\
\hline Barthel index & $45(20-70)$ & $50(20-65)$ & $65(20-95)$ & $20(0-50)$ \\
\hline \multicolumn{5}{|l|}{ Codon $129 \%$ (n) } \\
\hline $\mathrm{M} / \mathrm{M}$ & $0(0)$ & $20(1)$ & $25(14)$ & $24(8)$ \\
\hline $\mathrm{M} / \mathrm{V}$ & $43(3)$ & $40(2)$ & $40(22)$ & $39(13)$ \\
\hline V/V & $57(4)$ & $40(2)$ & $35(19)$ & $36(12)$ \\
\hline$\tau$ Protein & 2955 (1059-7984) & $2400(1200-8093)$ & 3320 (1095-5968) & $4700(2943-10805)$ \\
\hline Neuron-specific enolase & $107(25-204)$ & $73(49-97)$ & $30(21-41)$ & $61(36-120)$ \\
\hline Positive cases & Per cent & Per cent & Per cent & Per cent \\
\hline 14-3-3 positive & 94 & 92 & 78 & 95 \\
\hline PSWCs in EEG & 13 & 42 & 15 & 21 \\
\hline CJD-typical MRI & 83 & 92 & 89 & 75 \\
\hline Hyperintensities of basal ganglia & 79 & 75 & 81 & 70 \\
\hline Hyperintensities cortical & 31 & 53 & 31 & 53 \\
\hline
\end{tabular}

A

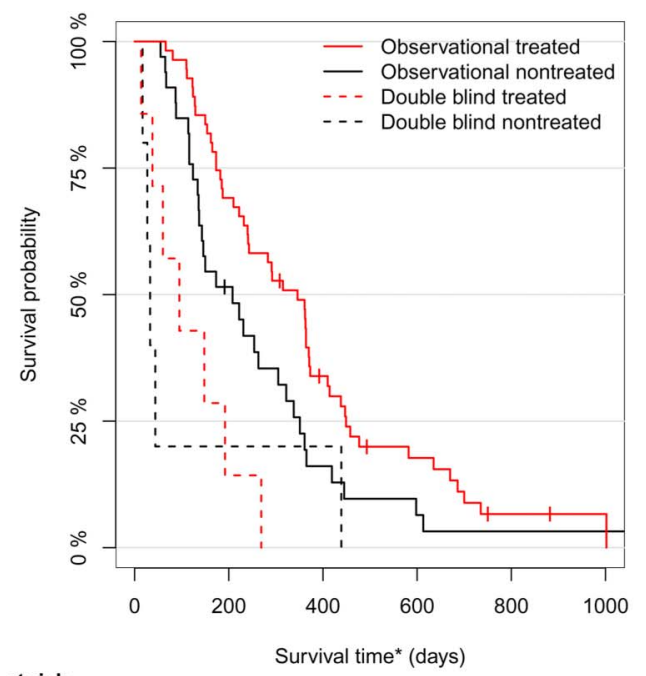

Patients at risk
Observational
treated
nontreated
Double blind
treated
nontreated

$\begin{array}{lllllllllll}55 & 53 & 38 & 29 & 17 & 9 & 8 & 4 & 2 & 1 & 1\end{array}$

$\begin{array}{lllllllllll}33 & 28 & 16 & 11 & 5 & 3 & 2 & 1 & 1 & 1 & 1\end{array}$

$\begin{array}{lllllllllll}7 & 3 & 1 & 0 & 0 & 0 & 0 & 0 & 0 & 0 & 0 \\ 5 & 1 & 1 & 1 & 1 & 0 & 0 & 0 & 0 & 0 & 0\end{array}$
B
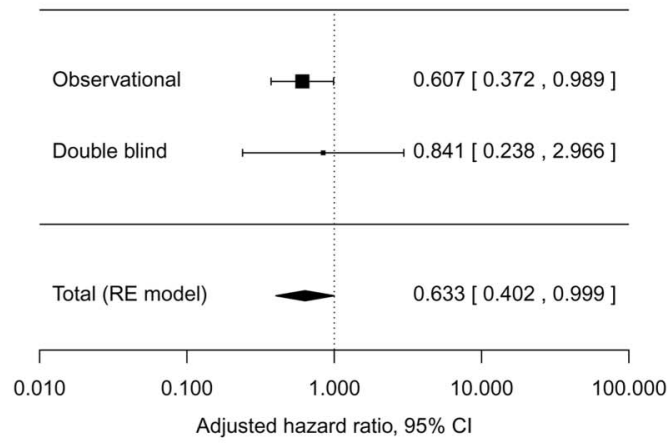

Adjusted hazard ratio, $95 \% \mathrm{Cl}$

\footnotetext{
*In the double blind trial follow-up for survival starts with the randomization whereas patients in the observational study are followed up for survival from first symptoms.
}

Figure 2 Survival times of doxycycline treated and non-treated patients. (A) Kaplan-Meier survival times of both trials. (B) Forest plot of the combined meta-analysis. RE, randomised effects.

were mainly dysphagia, respiratory insufficiency, infections of upper and lower airways, gastrointestinal disturbances, thromboses and urinary tract infections.

\section{The observational study}

Between May 2006 and March 2013, 850 patients with probable and definite sCJD were reported to the German TSE
Surveillance Center. Fifty-five were treated with doxycycline by their family doctors, and furthermore had a known codon 129 polymorphism status as well as known MMSE test results at the time of diagnosis. The mean age at onset of the doxycyclinetreated group was 62 years. In the neuropsychological examination, an MMSE of 20 points was achieved. The doxycycline therapy started 168 days from disease onset and the duration of 
Table 3 Secondary outcome measures, calculated according to Kaplan-Meier

\begin{tabular}{|c|c|c|c|c|c|c|c|c|}
\hline \multirow[b]{2}{*}{ Secondary outcome measures } & \multicolumn{4}{|c|}{ Double-blind study doxycycline } & \multicolumn{4}{|c|}{ Double-blind study control (placebo) } \\
\hline & BL & $1 \mathrm{TV}$ & 2TV & $8 W$ & BL & 1TV & 2TV & $8 W$ \\
\hline Probability of deficits in sphincter function (patients at risk) & $43 \%(7)$ & $62 \%(3)$ & $81 \%(1)$ & $81 \%(1)$ & $40 \%(5)$ & $80 \%(3)$ & $80 \%(1)$ & $80 \%(1)$ \\
\hline Probability of needing help in feeding/nutrition (patients at risk) & $71 \%(7)$ & $86 \%(2)$ & $86 \%(1)$ & $100 \%(1)$ & $80 \%(5)$ & $80 \%(1)$ & $80 \%(1)$ & $100 \%(1)$ \\
\hline Probability of akinetic mutism (patients at risk) & $0 \%(7)$ & $0 \%(6)$ & $33 \%(6)$ & $33 \%(2)$ & $0 \%(5)$ & $20 \%(5)$ & $20 \%(2)$ & $-(0)$ \\
\hline
\end{tabular}

1TV, First telephone interview 2 weeks after baseline; 2TV, second telephone interview 4 weeks after baseline; $8 \mathrm{~W}, 8$ weeks; BL, baseline.
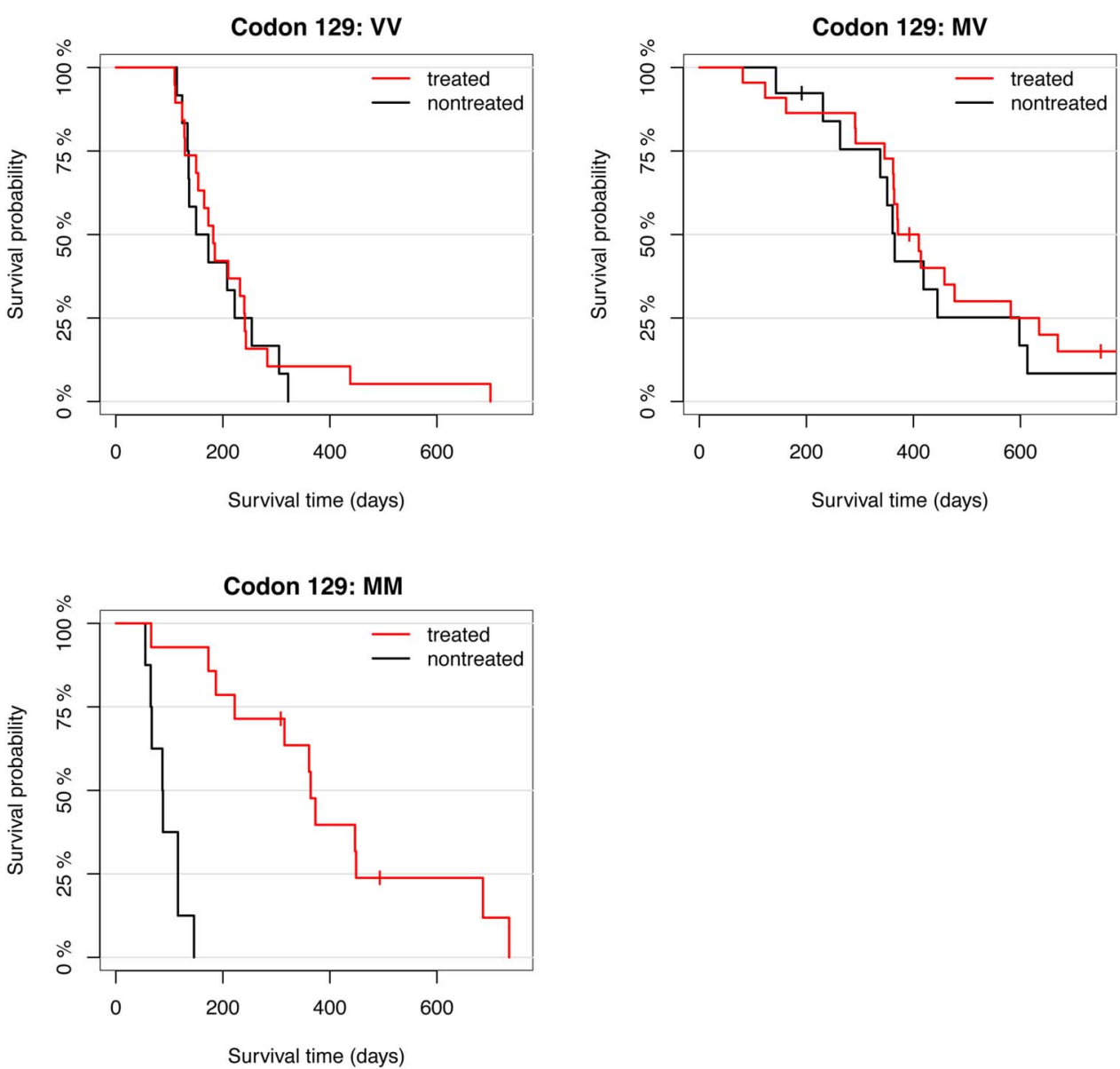

Figure 3 Influence of the codon 129 polymorphism on survival time in the observational study.

the doxycycline treatment was 122 days (mean). Thirty-three untreated patients with probable or definite SCJD with known codon 129 polymorphism genotype and MMSE from the same time period were analysed as controls. The clinical data and the spectrum of diagnostic findings with regard to biomarker in the cerebrospinal fluid, periodic sharp wave complexes in the EEG and increased signal intensity of the basal ganglia and/or the cortex in the MRI are described in detail in table 2. The influence of possible confounders (age of disease onset, gender, codon 129 genotype and baseline MMSE, according to ref. 16) has to be taken into account in the data analysis. The Cox regression was stratified by terciles of the corresponding propensity scores.

In the stratified analysis, we found a significant prolongation in survival time in patients treated with doxycycline compared with the untreated controls, with an estimated HR of 0.607 (95\% CI (0.372 to 0.989)). The (unadjusted) median survival time in the doxycycline-treated patients was 346 days compared with 208 days for the control group. The longer survival time in doxycycline-treated SCJD versus non-treated SCJD is shown in figure 2A. In both groups, the codon 129 genotype influenced the survival time in the doxycycline-treated group. The median survival time in the valine homozygous (VV) genotype without treatment is 162 days and with doxycycline therapy 182 days (HR 0.85). For the methionine/valine heterozygous (MV) genotype, the survival time is longer, with 365 days in the nontreated patients with sCJD and 391 days in treated sCJD (HR 0.83 ). The shortest survival time of 88 days was observed in a non-treated methionine homozygous (MM) patient with sCJD and the survival time in a doxycycline-treated patient with sCJD with this genotype was longer (364 days with a significant HR of 0.034). The Kaplan-Meier survival curves are shown in figure 3. Owing to the small sample sizes, stratification with a propensity score is not possible in this analysis and hence no 
adjustment for covariates was performed. Therefore, no $p$ values are reported for this analysis, underscoring the exploratory nature of these analyses.

\section{Combined analysis of both studies}

The results of both studies were formally combined in a random-effects meta-analysis resulting in a combined HR of 0.633 (95\% CI (0.402 to 0.999$)$ ) and demonstrating statistically significant superiority of doxycycline treatment over control $(\mathrm{p}=0.049$, z-test, random-effects meta-analysis). Here, the between-study heterogeneity was estimated to be 0 . The results are illustrated in the forest plot in figure $2 \mathrm{~B}$.

\section{DISCUSSION}

Only a few clinical trials on patients with CJD have been performed so far. Most data originate from anecdotal reports or observational trials. The first randomised trial was performed with flupirtine, ${ }^{11}$ which demonstrated the feasibility of a randomised trial in this devastating disease. Other placebo-controlled studies followed: quinacrine trials in the UK (PRION- $1^{17}$ ) and the USA ${ }^{18}$ and doxycycline in Italy and France. ${ }^{8}$ Although the results were negative, important lessons were learnt concerning recruitment protocols and study designs. The clinical scales developed in the PRION-1 trial ${ }^{17}$ are now under validation at several sites, including Germany, and the establishment of disease-specific scales to monitor disease progression in CJD will be an important contribution for further trials to come.

Future studies need to consider specific trial designs. ${ }^{19}$ The ultimate goal in a pharmacological intervention is the enrolment of patients at an early disease stage. Exactly this point was addressed in quinacrine trials and in our cohort. ${ }^{17} 18$ The experiences were similar-patients at early disease stages declined to receive placebo and did not agree to randomisation. With this, our study is in line with the observations made in the British and American US studies. Clearly, the classic standard double-blind placebo-controlled study design might be problematic for trial protocols in CJD and alternative concepts are needed.

We observed a prolongation of survival time in patients taking doxycycline. Our data differ from those reported by the multicentre trial. ${ }^{8}$ A potential explanation for the difference could be the disease stage when treatment was initiated. In our study, only patients at an early stage of the disease-those who could be tested by a battery of neuropsychological tests-were included. The assumption that the patients in our study were enrolled at an early stage is supported by the low frequency of PSWCs in the EEG which typically occurs in the middle and later stages. ${ }^{20}$ We are aware that our results might be biased by the small sample size in the double-blind trial as well as the inherent methodological problems of observational studies. On the other hand, single case reports demonstrate a similar phenomenon-extension of survival time when treatment starts early. $^{21} 22$

The influence of the codon 129 genotype (on clinical presentation with the shortest survival time in MM patients) is well established. ${ }^{23}$ Our results indicate a possible life-prolonging effect of a doxycycline therapy in MM patients. In contrast, valine homozygous and heterozygous patients displayed only a slight trend towards longer survival. The small patient numbers, which are also due to our strict inclusion criteria, might influence the statistical significance of our results. We cannot completely exclude that the observed effect might result from an antibiotic effect of doxycycline and thus from a general protective effect against infection. Nevertheless, the marked difference between the genotypes suggests a genotype-specific effect of the treatment. This finding additionally supports the assumption of a specific antiprion effect of doxycycline in contrast to an antibiotic effect, which would be similarly present in all genotypes.

To study the potential effect on the disease course, we evaluated when in the disease course important clinical hallmarks such as deficits in eating, mobility, speaking and sphincter control occur. Here, we found a significantly later appearance of these deficits in the doxycycline group. Therefore, we can assume an overall delay in disease progression, which is shown by the later decline in these abilities in treated patients.

Our patients' cohort displays an atypical distribution of codon 129 genotypes which might result from our selection criteria. We enrolled patients with only mild dementia, that is, those at an early stage of the disease. The MM disease subtype is typically characterised by very rapid dementia and is usually diagnosed at a late stage because of the aggressiveness of the disease. In contrast, in VV patients, ataxia is frequently the earliest clinical presentation, and dementia is mild at early stages. Consequently, these patients were more likely to be enrolled in our study.

On the basis of our results, we recommend doxycycline treatment, especially in MM patients with sCJD in earlier disease stages, as a useful therapeutic option, unless other treatment options become available.

Contributors DV was involved in study concept and design, and editing the manuscript. HM, CP, WJS-S, AK, MB and FF were involved in acquisition of data. UH was involved in study concept and acquisition of data. MS was involved in critical revision of the manuscript. KK and TF were involved in statistical analyses and critical revision of the manuscript. IZ was involved in study concept and supervision, and funding.

Funding This study was supported by the Federal Ministry of Education and Research (grant no. 01KG0905) and by the Robert Koch-Institute through funds from the Federal Ministry of Health (grant no. 1369-341). Some of the work by KK has received funding from FP HEALTH 2013 'Innovative methodology for small populations research' (InSPiRe) grant no. 602144.

Competing interests None declared.

\section{Patient consent Obtained.}

Ethics approval Ethikkommitee University of Goettingen.

Provenance and peer review Not commissioned; externally peer reviewed.

Open Access This is an Open Access article distributed in accordance with the Creative Commons Attribution Non Commercial (CC BY-NC 4.0) license, which permits others to distribute, remix, adapt, build upon this work non-commercially, and license their derivative works on different terms, provided the original work is properly cited and the use is non-commercial. See: http://creativecommons.org/ licenses/by-nc/4.0/

\section{REFERENCES}

1 Korth C, Peters PJ. Emerging pharmacotherapies for Creutzfeld-Jakob disease. Arch Neurol 2006;63:497-501.

2 Trevitt CR, Collinge J. A systematic review of prion therapeutics in experimental models. Brain 2006;129:2241-65.

3 Stewart LA, Rydzewska LH, Keogh GF, et al. Systematic review of therapeutic interventions in human prion diseases. Neurology 2008;70:1272-81.

4 Zerr I. Human prion diseases: progress in clinical trials. Brain 2013;136:996-7.

5 Appleby BS, Lyketsos CG. Rapidly progressive dementias and the treatment of human prion diseases. Expert Opin Pharmacother 2011;12:1-12.

6 Tagliavini F, Forloni G, Colombo L, et al. Tetracycline affects abnormal properties of synthetic PrP peptides and $\operatorname{PrP}(\mathrm{Sc})$ in vitro. J Mol Biol 2000;300:1309-22.

7 Forloni G, Salmona M, Marcon G, et al. Tetracyclines and prion infectivity. Infect Disord Drug Targets 2009;9:23-30.

8 Haïk S, Marcon G, Mallet A, et al. Doxycycline in Creutzfeldt-Jakob disease: a phase 2, randomised, double-blind, placebo-controlled trial. Lancet Neurol 2014;13:150-8.

9 WHO. Human transmissible spongiform encephalopathies. Wkly Epidemiol Rec 1998; 47:361-5

10 Zerr I, Kallenberg K, Summers DM, et al. Updated clinical diagnostic criteria for sporadic Creutzfeldt-Jakob disease. Brain 2009;132:2659-68.

11 Otto M, Cepek L, Ratzka P, et al. Efficacy of flupirtine on cognitive function in patients with CJD: a double-blind study. Neurology 2004;62:714-18. 
12 Varges D, Jung K, Gawinecka J, et al. Amyloid-b 1-42 levels are modified by apolipoprotein E e4 in Creutzfeldt-Jakob disease in a similar manner as in Alzheimer's disease. J Alzheimers Dis 2011;23:717-26.

13 Zerr I, Pocchiari M, Collins S, et al. Analysis of EEG and CSF 14-3-3 proteins as aids to the diagnosis of Creutzfeldt-Jakob disease. Neurology 2000;55: 811-15.

14 Windl 0, Giese A, Schulz-Schaeffer W, et al. Molecular genetics of human prion diseases in Germany. Hum Genet 1999;105:244-52.

15 Higgins JP, Thompson SG, Deeks JJ, et al. Measuring inconsistency in metaanalyses. BMJ 2003:327:557-60

16 Ladogana A, Puopolo M, Croes EA, et al. Mortality from Creutzfeldt-Jakob disease and related disorders in Europe, Australia, and Canada. Neurology 2005:64:1586-91.

17 Collinge J, Gorham M, Hudson F, et al. Safety and efficacy of quinacrine in human prion disease (PRION-1 study): a patient-preference trial. Lancet Neurol 2009:8:334-44.

18 Geschwind MD, Kuo AL, Wong KS, et al. Quinacrine treatment trial for sporadic Creutzfeldt-Jakob disease. Neurology 2013;81:2015-23.

19 Mead S, Burnell M, Lowe J, et al. Clinical trial simulations based on genetic stratification and the natural history of a functional outcome measure in Creutzfeldt-Jakob disease. JAMA Neurol 2016;73:447-55.
20 Wieser HG, Schindler K, Zumsteg D. EEG in Creutzfeldt-Jakob disease. Clin Neurophysiol 2006;117:935-51.

21 Assar H, Topakian R, Weis S, et al. A case of variably protease-sensitive prionopathy treated with doxycyclin. J Neurol Neurosurg Psychiatry 2015;86:816.

22 Pocchiari M, Ladogana A. Rethinking of doxycycline therapy in Creutzfeldt-Jakob disease. J Neurol Neurosurg Psychiatry 2015;86:705.

23 Zerr I. Therapeutic trails in human transmissible spongiform encephalopaths: recent advances and problems to address. Infect Disord Drug Targets 2009:1:92-9.

24 Terzano MG, Montanari E, Calzetti S, et al. The effect of amantadine on arousal and EEG patterns in Creutzfeldt-Jakob disease. Arch Neurol 1983;40:555-9.

25 Neri G, Figa TL, Di BG, et al. [Amantadine in Creutzfeldt-Jakob disease. Review of the literature and case contribution]. Riv Neurobiol 1984;30:47-56.

26 Bone I, Belton L, Walker AS, et al. Intraventricular pentosan polysulphate in human prion diseases: an observational study in the UK. Eur J Neurol 2008;15:458-64

27 Tsuboi Y, Doh-Ura K, Yamada T. Continuous intraventricular infusion of pentosan polysulfate: clinical trial against prion diseases. Neuropathology 2009;29:632-6.

28 Haïk S, Brandel JP, Salomon D, et al. Compassionate use of quinacrine in Creutzfeldt-Jakob disease fails to show significant effects. Neurology 2004;63:2413-15. 